山्山FANÇAISE

$>\mathrm{DE}$

$\stackrel{1=1}{\simeq}$ PÉDAGOGIE

\section{Revue française de pédagogie}

Recherches en éducation

156 | juillet-septembre 2006

Les espaces locaux d'interdépendance entre

établissements : une comparaison européenne

\title{
FRANÇOIS Frédéric. Interprétation et dialogue chez des enfants et quelques autres : recueil d'articles
}

1988-1995

Lyon : Éd. de l'ENS, 2005. - 288 p. (Langages).

Lucie Mottier-Lopez

\section{OpenEdition \\ Journals}

\section{Édition électronique}

URL : http://journals.openedition.org/rfp/661

DOI : $10.4000 /$ rfp.661

ISSN : 2105-2913

Éditeur

ENS Éditions

Édition imprimée

Date de publication : 1 juillet 2006

Pagination : 182-184

ISBN : 978-2-7342-1060-3

ISSN : 0556-7807

\section{Référence électronique}

Lucie Mottier-Lopez, « FRAnçoıs Frédéric. Interprétation et dialogue chez des enfants et quelques autres : recueil d'articles 1988-1995 », Revue française de pédagogie [En ligne], 156 | juillet-septembre 2006, mis en ligne le 24 septembre 2010, consulté le 25 septembre 2020. URL : http://

journals.openedition.org/rfp/661 ; DOI : https://doi.org/10.4000/rfp.661

Ce document a été généré automatiquement le 25 septembre 2020.

(c) tous droits réservés 


\section{FRANÇOIS Frédéric. Interprétation et dialogue chez des enfants et quelques autres : recueil d'articles 1988-1995}

Lyon : Éd. de l'ENS, 2005. - 288 p. (Langages).

Lucie Mottier-Lopez

\section{RÉFÉRENCE}

FRANÇOIS Frédéric. Interprétation et dialogue chez des enfants et quelques autres :

recueil d'articles 1988-1995. Lyon : Éd. de l'ENS, 2005. - 288 p. (Langages).

1 L'ouvrage réunit un ensemble d'articles publiés entre 1988 et 1995 dont le trait commun est d'interroger sans relâche la relation entre le dialogue et l'interprétation. L'auteur réfute l'idée d'une " grammaire des enchaînements » qui seule caractériserait le dialogue; il argumente, avec force et moult « mouvements », l'interprétation comme dialogisme. Ce livre témoigne de la pensée dialogique de François peut-être d'abord avec lui-même, dans ses détours, ses interrogations, ses exemples, ses références.

Dans une longue introduction de trente-sept pages, François propose un éclairage «actuel» (fin 2004) de ses articles dans une logique, dit-il, de "continuitédéplacement ». Il prend comme point de départ la question du sens qui comporte à la fois un côté «fermé » - un assignable, un dominant - et un côté " ouvert » avec des entours plus ou moins partagés, labiles, dicibles, des champs de possibilités, de ressemblances, d'interrogations... entre constance et variance, entre unité et diversité, toujours dans un processus d'interprétation dialogique. Ce faisant, l'auteur introduit des premières oppositions parmi de nombreuses autres dichotomies qu'il n'aura cesse, ensuite, de discuter et de remettre en question tout au long de son livre: physique/ mental, cognition/affect, réel/irréel, générique/particulier, interne/externe, continu/ 
variable, quotidien/scientifique, partagé/non partagé/supposé partagé... Le ton est donné : l'auteur nous livre sa pensée non dualiste et relativiste, avec le paradoxe peutêtre que, pour ce faire, il s'appuie constamment sur des oppositions qu'il remet en cause mais dont il reconnaît pourtant une forme d'utilité et de permanence pour «penser le sens ». Si les objets d'étude premiers de François sont les discours et les textes, ses développements valent également pour les façons d'être et d'agir des humains vues comme foncièrement liées à des dynamiques d'interprétation, constitutives et constituées par des contextes et des individus pluriels. C'est sans conteste un des points forts de cet ouvrage qui s'adresse à toute personne intéressée par la question du sens.

3 Les termes qui caractérisent la pensée de l'auteur sont discutés dans l'introduction: interprétation, dialogue, mouvements, régimes de signification pour les principaux. L'objectif est de « contribuer à éclairer ce que c'est qu'un texte comme mouvement et non comme structure, à justifier aussi l'impossibilité de dépasser la pluralité des points de vue » (p. 42). Très dense, voire difficile d'accès pour des lecteurs non spécialistes, cette introduction prend plus particulièrement sens (!) une fois lus les différents textes réunis dans l'ouvrage, dans un va-et-vient dialogique que l'auteur ne renierait pas. Les textes réunis sont organisés en trois parties: 1) Remarques méthodologiques; 2) L'organisation du discours et le langage de l'enfant; 3) Langage et "discours pathologique ».

4 Les cinq textes qui composent la première partie explicitent le point de vue méthodologique de l'auteur. Ce dernier s'oppose à l'idée de prendre comme modèle les sciences de la nature pour étudier l'humain, la langue en particulier. Le but, argue-t-il, n'est pas de dégager une «loi de la langue » mais d'appréhender les procédures de variation par lesquelles «les façons de faire sens se modifient dans le cours même du discours et de sa réception » (p. 42). Ce point de vue est spécialement développé dans le premier texte « Pourquoi change-t-on de théorie ? Quelques remarques sur les sciences de l'homme en général et la linguistique en particulier ». Dans le deuxième texte "Quelques remarques sur la notion d'interprétation ou du dialogue quotidien comme modèle d'interprétation", l'auteur argumente la nature dialogique du terme même d'interprétation en présentant, notamment, un ensemble de critères qui permettraient de caractériser, ou non, un discours comme interprétation. Il s'agit d'un apport majeur de ce texte. Deux exemples de dialogue sont ensuite donnés, l'un entre des enfants de CP et l'autre entre des adolescents de 17-18 ans. L'analyse interprétative de l'auteur, effectivement sous-tendue par une logique foncièrement dialogique, donne à voir sa conception de l'interprétation en tant que mouvements signifiants de déplacementmodification; quelques conditions à l'interprétation sont énoncées. Les deux textes suivants «Le 'signifié' ou les types de mise en mots" et "Sémantiques et significations " traitent essentiellement la question de la signification, des jeux de langage et des variations sémiologiques. L'auteur clôt cette partie méthodologique par le texte "Langage et pensée: dialogue et mouvement discursif chez Vygotski et Bakhtine ». Quelques thèses vygotskiennes sont relues à la lumière de la pensée de Bakhtine - dont l'auteur se dit proche, notamment de l'idée de compréhension responsive - afin de souligner, entre autres, les relations diversifiées entre langage et expériences. Le projet scientifique de l'auteur se dégage : développer une «théorie des mouvements discursifs " plutôt que des structures de la langue, une théorie qui amène à questionner la relation entre le dialogue et la pensée (et non la langue et la pensée). Si ce texte, court, commence en soulignant le dialogue inégal qui lie l'enfant et l'adulte, par 
exemple dans la zone proximale de développement, on peut regretter que l'exemple concret donné par François ne reprenne pas des analyses d'échanges précisément asymétriques pour questionner ce lien qualifié de consubstantiel entre dialogue et pensée et pour montrer que le discours de chacun constitue un mouvement par rapport au discours de l'autre. À la fin de cette première partie "méthodologique ", on s'impatiente presque d'entrer dans davantage d'exemples d'analyses de corpus afin, comme le dit l'auteur, de saisir finement comment des mêmes mots utilisés peuvent, de fait, renvoyer à des significations et à des réalités différentes.

La deuxième partie de l'ouvrage regroupe quatre textes qui développent l'analyse du langage des enfants. Le premier "Continuité et mouvements discursifs. Un exemple chez des enfants de trois ans " se centre sur un aspect du dialogue en particulier : les significations portées non pas par les messages en tant que tels mais par leurs enchaînements locaux. L'auteur évoque, dans un premier temps, les problèmes que pose ce type d'analyse et il souligne les « dangers méthodologiques » notamment dans le choix des exemples donnés à voir. Il explicite sa posture : « un dialogue constitue un événement et non pas simplement un cas particulier qui illustrerait la généralité de la 'loi'" , « il n'y a pas de liens directs entre degré de généralité et degré de scientificité », «il s'agit plus de montrer que de démontrer» (p. 147). François expose, dans un deuxième temps, les procédures d'analyse d'un dialogue entre deux petites filles en présence de deux adultes. Une analyse en quatre points est proposée: 1) la détermination des unités ; 2) les facteurs de communauté entre les interlocuteurs ; 3) les facteurs de distance, d'événementialité ou d'irruption ; 4) les types de significations, organisateurs dominants. Le deuxième texte "Stratégies discursives chez les enfants. Un exemple : les définitions " poursuit la (dé)monstration de la pluralité des façons de mettre en mots la « réalité » selon les variations des objets de discours et des conditions d'interlocution notamment. En s'appuyant sur des propos de jeunes enfants, l'auteur a pour projet de montrer qu'il n'y a pas «une» logique naturelle mais des stratégies discursives différentes, qu'il y a des mots « unificateurs » qui sont définis plus ou moins de la même manière et des mots "différenciateurs", que les enfants pensent par reprises, déplacements, modalisations comme le font les adultes. L'auteur donne des exemples de définition de mots proposées par des enfants afin d'illustrer et de questionner ces différents points. La diversité du rapport des enfants aux mots est soulignée. De même, il est montré qu'on ne parle/pense pas de la même façon quand on est seul ou quand on est en relation avec le discours d'autrui. Comme l'indique le titre du troisième texte «Le corps comme langage, l'oral, l'écrit. Interactions et conflits sémiotiques ", les relations multiples entre l'oral et l'écrit et entre la signification et le corps sont ensuite au centre du discours de François. Celui-ci livre quelques éléments de sa conception de l'entrée dans le langage du petit enfant : il ne s'agit plus de partir du premier mot prononcé par l'enfant mais de partir d'éléments déjà là, fondateurs de la sémiose, telle que l'existence de sens inscrit dans le monde extérieur et les objets culturels, dans les gestes corporels, dans les expériences et les rapports à autrui. La relation de fermeture-ouverture est discutée dans le rapport de l'écrit à l'oral. Finalement, le dernier texte qui clôt la deuxième partie du livre "Voir et dire: convergences et divergences, mouvements discursifs et effets de sens ", met en avant quelques homologies et quelques contrastes entre la mise en signes par le dessin et un dialogue entre une enseignante et ses élèves de grande section maternelle à propos de ces mêmes dessins. Pour effectuer cette comparaison, un ensemble de notions sont introduites, dont celles de résidu, significations locales/significations atmosphériques, 
facile/difficile à dire, discours et événements, culture, altérité et imaginaire. Quatre exemples sont donnés et l'auteur de commenter : " on a essayé de n'être ni linguiste ni psy... pas linguiste dans la mesure où ce qui importe ici ce n'est pas tant les mots ou les structures utilisées... mais au contraire leur façon de faire effet dans une situation particulière, en particulier par leur relation de déplacement par rapport au discours de l'autre ou de soi » (p. 207). De façon un peu provocante, on a envie de rétorquer à l'auteur: alors de quel type d'analyse «scientifique » s'agit-il ? Et ce dernier de nous répondre : «j'ai cherché à systématiser un peu les effets du dialogue second que j'ai eu avec ces dialogues... j'espère que ce dialogue produira sur les lecteurs des effets de dialogue productifs, polémiques et/ou inattendus dans leurs propres relations aux mises en mots des enfants » (p. 207). Autrement dit, l'objectif est d'inciter les lecteurs/ récepteurs à construire une relation dialogique et interprétative aux paroles des enfants - et donc immanquablement variable d'un récepteur à l'autre selon ses propres cadres interprétatifs. Pour ma part, l'objectif est atteint, et certainement plus encore dans mon rapport questionnant aux interprétations mêmes que l'auteur expose des dialogues des enfants. Extrêmement intéressants, on aurait aimé qu'il y ait davantage d'exemples de ce type dans cette partie du livre qui reste essentiellement méthodologique et théorique.

6 Finalement, la dernière partie de l'ouvrage est composée de deux textes qui proposent une réflexion sur le langage dans ce que l'on appelle la "pathologie mentale». Le premier texte «De quelques aspects du dialogue psychiatre-patient. Places, genres, mondes et compagnie " revient sur l'analyse des mouvements discursifs et discute différents aspects des dialogues médecins-malades. Le deuxième texte "Perceval, Schreber, Rousseau: écrire sa folie» interroge la façon dont est mise en mots la «folie» par ces trois psychotiques célèbres. Les situations sont évidemment foncièrement différentes des dialogues d'enfants exposés précédemment. Mais le thème est récurrent : celui de l'interprétation comme dialogisme.

Globalement, les apports de l'ouvrage sont nombreux, notamment dans l'appréhension de la diversité des régimes de signification tels qu'ils se dessinent dans le déroulement des échanges, dans le rapport à soi et aux autres, dans le rapport aux personnes présentes, absentes, imaginaires, dans le rapport aux mots, aux objets culturels, au monde. François invite à questionner tout spécialement la façon dont les enfants s'approprient le langage. Il n'esquisse cependant pas d'implications pédagogiques et didactiques. Tel n'est d'ailleurs pas le projet de l'auteur. Très denses, certains passages ne sont pas d'un abord facile. Une conséquence peut-être de la forme du livre: un recueil de textes pour la plupart courts mais qui portent sur des champs thématiques larges et complexes. L'auteur le reconnaît lui-même : ses développements peuvent être perçus comme redondants. Cette redondance représente aussi une qualité pour qui veut entrer dans la pensée philosophique - et pas seulement linguistique - de l'auteur. Un éclairage par touches successives est ainsi offert qui donne envie de relire certains textes, de reconstruire le livre dans un ordre personnel en fonction de son propre dialogue interprétatif avec François, avec les personnes qu'il convoque et avec soimême. Dans la lignée des propos de l'auteur, terminons en soulignant que cette note critique est évidemment le produit d'une interprétation subjective des textes du livre; une autre lecture aurait pu en être faite. À chacun donc de se laisser entraîner par sa propre lecture interprétative de l'ouvrage. 


\section{AUTEURS}

LUCIE MOTTIER-LOPEZ

Université de Genève 